\title{
Erratum to: The OECD validation program of the H295R steroidogenesis assay: Phase 3. Final inter-laboratory validation study
}

\author{
Markus Hecker $^{1,2}$ • Henner Hollert ${ }^{3} \cdot$ Ralph Cooper $^{4}$ - Anne-Marie Vinggaard ${ }^{5}$. \\ Yumi Akahori $^{6}$ - Margaret Murphy ${ }^{7}$. Christine Nellemann ${ }^{5}$. Eric Higley ${ }^{2}$. \\ John Newsted ${ }^{8}$ • John Laskey ${ }^{9}$ - Angela Buckalew ${ }^{4}$ - Stefanie Grund ${ }^{10}$ • Sibylle Maletz ${ }^{3}$. \\ John Giesy ${ }^{2,7} \cdot$ Gary Timm $^{11}$
}

Published online: 23 October 2017

(C) Springer-Verlag GmbH Germany 2017

Erratum to: Environ Sci Pollut Res (2011) 18: 503 https://doi.org/10.1007/s11356-010-0396-x

In the original article wrong unites were quoted in Table 3 (page 508) and Table 4 (page 510) as well as in the paragraph 3.2 Core chemical exposure experiments on page 509. Also in paragraph 2.3 Selection and testing of chemicals the link to the Supplemental Materials (ESM) was missing. The correct versions of the tables and the paragraph as well as the ESM link are provided below.

The online version of the original article can be found at https://doi.org/ 10.1007/s11356-010-0396-x

Electronic supplementary material The online version of this article (https://doi.org/10.1007/s11356-017-0321-7) contains supplementary material, which is available to authorized users.

Markus Hecker

markus.hecker@usask.ca

ENTRIX, Inc., Sasaktoon, SK S7N 5B3, Canada

2 Department of Veterinary Biomedical Sciences and Toxicology Centre, University of Saskatchewan, Saskatoon,

Saskatchewan, Canada

3 Department of Ecosystem Analysis, Institute for Environmental Research, RWTH Aachen University, 52074 Aachen, Germany

4 Endocrinology Branch, RTD, NHEERL, ORD, US Environmental Protection Agency, Research Triangle Park, NC, USA

5 Department of Toxicology \& Risk Assessment, National Food Institute, Technical University of Denmark, Soborg, Denmark

\subsection{Core chemical exposure experiments}

There were chemical-specific differences in the response of $\mathrm{T}$ production after exposure of $\mathrm{H} 295 \mathrm{R}$ cells to the 12 core chemicals (Table 3 ). With a few exceptions, the observed chemical-specific responses of $\mathrm{T}$ production were comparable among laboratories and could be grouped into three different types of effects: inducers, inhibitors, and negative reference chemicals. Among the inducers, exposure to trilostane resulted in the greatest fold changes ( $>10$-fold induction) in T concentra-

6 Chemicals Evaluation and Research Institute, Chemicals Assessment Center, Saitama, Japan

7 Department of Biology \& Chemistry, City University of Hong Kong, Kowloon, Hong Kong, SAR, China

8 ENTRIX, Inc., Okemos, MI 48864, USA

9 Senior Environmental Employment Program, National Caucus on Black Aged, Research Triangle Park, NC, USA

10 Department of Zoology, University of Heidelberg, 69120 Heidelberg, Germany

11 Office of Science Coordination \& Policy, US Environmental Protection Agency, Washington, DC, USA 
tion when compared to SCs. The least fold changes were observed for the atrazine exposures where induction of $\mathrm{T}$ production was less than 1.5 -fold with the exception of Lab 2, at which maximum induction was 2.4 -fold. No effect on T production was observed after exposure to atrazine at Lab 6. Exposure to prochloraz resulted in a greater than 15 -fold reduction of $\mathrm{T}$ production at the greatest concentration tested $(100 \mu \mathrm{M})$ at all laboratories with the exception of Lab 4 where an up to 4.5 -fold reduction was observed. The greater LOEC reported for Lab 2 is likely a function of the relatively great variation among replicate experiments at $0.01 \mu \mathrm{M}(\mathrm{CV}=35 \%)$. It is unclear why $\mathrm{T}$ production by cells was more sensitive to the exposure with prochloraz at Labs 1 and 3. However, a concentration-dependent response was observed starting at $0.01 \mu \mathrm{M}$, which is similar to the response patterns at the other labs. Therefore, it cannot be excluded that the significant reduction at 0.0001 and $0.001 \mu \mathrm{M}$ represents an artifact. Exposure to the other inhibitors resulted in less than 4-fold changes in $\mathrm{T}$ production. When chemicals exhibited a less than 1.5 -fold change in $\mathrm{T}$ production, they were categorized

Table 3 Lowest observed effect concentrations (LOECs; measured by Dunnett's or Mann Whitney $U$ test ${ }^{\mathrm{mu}}$ ) and strength and direction of change $(\Downarrow=>0.5$-fold; $\Downarrow \Downarrow=0.5$ - to $>0.25$-fold; $\Downarrow \Downarrow \Downarrow=0.25$ - to $>0.1$ fold; $\Downarrow \Downarrow \Downarrow \Downarrow=\leq 0.1$-fold; $\Uparrow=<2$-fold; $\Uparrow \Uparrow=2$ - to $<$ fold; $\Uparrow \Uparrow \Uparrow=4$ - to $<20$-fold; $\Uparrow \Uparrow \Uparrow \Uparrow=\geq 20$-fold) for testosterone (T) and estradiol (E2) after as negatives. This threshold was defined based on the average variation observed across all laboratories among replicate experiments. Some of these negative chemicals could have been categorized as inhibitors in individual cases (molinate: Lab 4; benomyl: Lab 1). However, even in situations where inhibition was observed at an individual laboratory, changes were always less than 2-fold and typically were not concentration-dependent. For instance, exposure to nonoxynol-9 resulted in a decrease in $\mathrm{T}$ concentrations at non-cytotoxic concentrations at two of five laboratories for which data was available. Relative to the SCs, inhibition of $\mathrm{T}$ production at Lab 1 was $29 \%(1 \mu \mathrm{M})$, while at Lab 2, it was $47 \%(10 \mu \mathrm{M})$. However, it should be noted that, at Lab 2, exposure to $10 \mu \mathrm{M}$ nonoxynol-9 resulted in an average increase in cell viability ( $138 \%$ viable cells relative to the SCs), and thus the observed reduction in $\mathrm{T}$ production may be an artifact due to the correction for cell viability, especially as no such increase was observed by any of the other groups. The greatest letrozole concentration resulted in a significant decrease in $\mathrm{T}$ at all laboratories.

exposure to the twelve core chemicals. Ranges refer to maximum values measured in repeated experiments. nd - not detectable; — chemical not analyzed. Gray shaded cells - uncertainty due to interference of the antibody based hormone detection system with the test chemical

\begin{tabular}{|c|c|c|c|c|c|c|c|c|c|c|}
\hline & \multicolumn{10}{|c|}{ Fold-Change (Testosterone) } \\
\hline & $\operatorname{Lab} 1^{a}$ & & Lab 2 & & Lab 3 & & Lab 4 & & Lab 6 & \\
\hline & LOEC $[\mu M]$ & Max Change & $L O E C[\mu M]$ & Max Change & LOEC $[\mu M]$ & Max Change & $\operatorname{LOEC}[\mu \mathrm{M}]$ & Max Change & $\operatorname{LOEC}[\mu \mathrm{M}]$ & Max Change \\
\hline Prochloraz & 0.0001 & Лூூூ & 0.1 & Лூூூ ூ & 0.0001 & Лூூூ & 0.01 & Лூூூ & 0.01 & $\sqrt{2} \sqrt{3}$ \\
\hline Aminoglutethimide & $100^{\mathrm{d}}$ & Лூூ & $100^{\mathrm{d}}$ & ת囚 & 10 & Лூת & $100^{\mathrm{d}}$ & $\sqrt{\Omega}$ & $100^{\mathrm{d}}$ & Лூூ \\
\hline Letrozole & $100^{\mathrm{d}}$ & Лת & $100^{d}$ & ת ת & $100^{a, d}$ & Лת & $100^{\mathrm{d}}$ & ת囚 & $100^{\mathrm{d}}$ & תู \\
\hline Nonoxynol-9 & $10^{\mathrm{c}, \mathrm{d}}$ & $\sqrt{ }$ & $10^{c, d}$ & $\sqrt{3}$ & $n d^{e}$ & & $10^{\mathrm{c}, \mathrm{d}}$ & $\sqrt{2}$ & $10^{c, d}$ & $\sqrt{2}$ \\
\hline Molinate & nd & & nd & & 100 & $\sqrt{3}$ & nd & & nd & \\
\hline Benomyl & nd & & nd & & nd & & $\mathrm{nd}^{m u}$ & & nd & \\
\hline EDS & nd & & nd & & nd & & nd & & nd & \\
\hline HCG & nd & & nd & & nd & & nd & & nd & \\
\hline Paraben & 10 & 仓 & nd & & 1 & 仓 & nd & & nd & \\
\hline Atrazine & $100^{d}$ & 仓 & 1 & 仓 & $100^{d}$ & 仓 & nd & & nd & \\
\hline Forskolin & 10 & 仓仓 & 1 & 仓仓 & 1 & 仓 & 1 & 仓仓 & 1 & 仓仓 \\
\hline \multirow[t]{4}{*}{ Trilostane } & $0.1^{m u}$ & 仓仓仓 & $0.01^{m u}$ & 仓仓仓 & $1^{m u}$ & 仓仓仓仓 & $1^{m u}$ & 仓仓仓仓 & $0.01^{m u}$ & 仓仓仓仓 \\
\hline & \multicolumn{10}{|c|}{ Fold-Change (Estradiol) } \\
\hline & $\operatorname{Lab} 1^{a}$ & & Lab 2 & & Lab 3 & & Lab 4 & & Lab 6 & \\
\hline & $\operatorname{LOEC}[\mu \mathrm{M}]$ & Max Change & LOEC $[\mu M]$ & Max Change & LOEC $[\mu M]$ & Max Change & $\operatorname{LOEC}[\mu \mathrm{M}]$ & Max Change $L$ & LOEC $[\mu \mathrm{M}]$ & Max Change \\
\hline Letrozole & 0.001 & $\sqrt{2}$ & 0.001 & תู & $0.0001^{m u}$ & Лூ囚 & 0.01 & Лூת & 0.01 & Лת \\
\hline Prochloraz & 0.1 & గ几 & 1 & $\sqrt{3}$ & 0.1 & $\sqrt{\sqrt{3}}$ & 1 & Лת & 0.1 & $\sqrt{2}$ \\
\hline Aminoglutethimide & $100^{d}$ & $\sqrt{\Omega}$ & $10^{m u}$ & 凤ת & 10 & Л』 & $100^{b, d}$ & Л』 & $100^{d}$ & Лת \\
\hline Benomyl & nd & & nd & & $\mathrm{nd}^{\mathrm{a}}$ & & $\mathrm{nd}^{\mathrm{a}}$ & & nd & \\
\hline EDS & nd & & nd & & nd & & nd & & nd & \\
\hline Nonoxynol-9 & nd & & nd & & nd & & nd & & nd & \\
\hline HCG & nd & & nd & 仓े & $\mathrm{nd}^{\mathrm{a}}$ & & $\mathrm{nd}^{\mathrm{a}}$ & & nd & \\
\hline Paraben & $\mathrm{nd}^{m u}$ & 仓仓仓 & 10 & 仓 & $10^{m u}$ & 仓仓 & nd & 仓ิ & nd & \\
\hline Molinate & $100^{d}$ & 仓仓 $m u$ & $100^{d}$ & 仓े & $100^{\mathrm{d} \boldsymbol{m} u}$ & 仓仓 & $100^{\mathrm{d} m u}$ & 仓仓 & $100^{d}$ & 仓仓 \\
\hline Atrazine & 10 & 仓仓仓 & $1^{m u}$ & 仓仓仓 & $1^{m u}$ & 仓仓仓仓 & $10^{m u}$ & 仓仓仓 & 0.1 & 仓 \\
\hline Forskolin & $0.01^{m u}$ & 仓仓仓仓 & $0.1^{m u}$ & 仓仓仓仓 & $0.1^{m u}$ & 仓仓仓仓 & $0.1^{m u}$ & 仓仓仓仓 & $0.01^{m u}$ & 仓仓 \\
\hline Trilostane & $1^{m u}$ & 仓仓仓仓 & 100 & 仓 & $0.1^{m u}$ & 仓仓仓仓 & $1^{m u}$ & 仓仓仓仓 & $1^{m u}$ & 仓仓仓 \\
\hline
\end{tabular}

${ }^{\mathrm{a}}$ Only one experiment was conducted or considered for data evaluation

${ }^{\mathrm{b}}$ Not statistically significant; $p=0.051$

${ }^{\mathrm{c}}$ Greatest concentration cytotoxic

${ }^{\mathrm{d}}$ Effects occurred at greatest non-cytotoxic concentration; no dose-response

${ }^{\mathrm{e}}$ Cytotoxicity observed at concentration at which effects occurred at other laboratories $=10$ 
Table 4 Lowest observed effect concentrations (LOECs; measured by Dunnett's test) and strength and direction of change ( $\Downarrow=>0.5$-fold; $\Downarrow \Downarrow=$ 0.5 - to $>0.25$-fold; $\Downarrow \Downarrow \Downarrow=0.25$ - to $>0.1$-fold; $\Downarrow \Downarrow \Downarrow \Downarrow=\leq 0.1$-fold; $\Uparrow=<2$ fold; $\Uparrow \Uparrow=2$ - to $<$ fold; $\Uparrow \Uparrow \Uparrow=4$ - to $<20$-fold; $\Uparrow \Uparrow \Uparrow \Uparrow=\geq 20$-fold) observed for the 16 test chemicals. nd - not detectable. Chemicals tested at the second laboratories (2nd Lab) were as follows; Lab2: Piperonyl butoxide, ketoconazole, prometon, DEHP, flutamide and danazol; Lab3: Bisphenol A, fenarimol, genistein, finasteride and dinitrophenol; Lab4: Spironolactone, mifepristone, tricrecyl phosphate, dimethoate and glyphosate

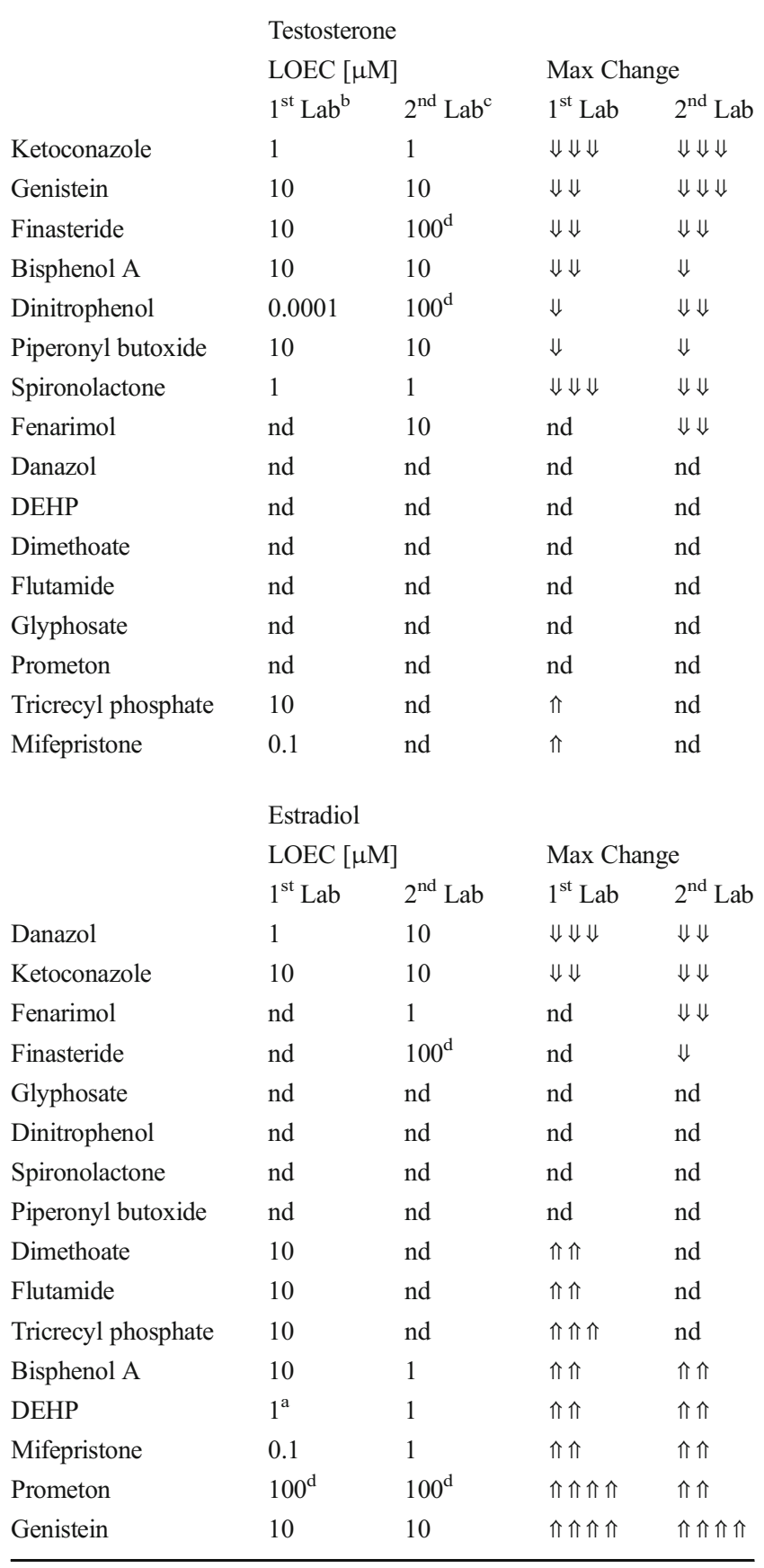

${ }^{a}$ considered because there was a clear concentration-response at all but the greatest concentration

${ }^{\mathrm{b}}$ lead laboratory (Lab 1)

${ }^{\mathrm{c}}$ participating laboratory (Labs 2,3 and 4)

${ }^{\mathrm{d}}$ Effects occurred at greatest non-cytotoxic concentration; no dose-response 